\title{
Circadian variation of heart rate is affected by environment: a study of continuous electrocardiographic monitoring in members of a symphony orchestra
}

\author{
D Mulcahy, J Keegan, A Fingret, C Wright, A Park, J Sparrow, D Curcher, K M Fox
}

\begin{abstract}
Twenty four hour ambulatory ST segment monitoring was performed on 48 members (43 players and five members of the management/technical team) of the British Broadcasting Corporation (BBC) symphony orchestra without a history of cardiac disease. This period included final rehearsals and live performances (for audience and radio) of music by Richard Strauss and Mozart at the Royal Festival Hall $(n=36)$ and Rachmaninov and Tchaikovsky at the Barbican Arts Centre $(n=21)$. During the period of monitoring one person (2\%) had transient ST segment changes. Mean heart rates were significantly higher during the live performances than during the rehearsals. Mean heart rates during the live performance of Rachmaninov and Tchaikovsky were significantly higher than during Strauss and Mozart in those $(n=6)$ who were monitored on both occasions. Mean heart rates in the management and technical team were higher than those of the players. The recognised circadian pattern of heart rate, with a peak in the morning waking hours, was altered similarly during both concert days, with a primary peak occurring in the evening hours and a lesser peak in the morning for both musicians and management/ technical staff.
\end{abstract}

This study showed that environmental factors are of primary importance in defining the circadian pattern of heart rate. This has important implications when identifying peak periods of cardiovascular stress and tailoring drug treatment for patients with angina pectoris.

The morning waking hours are the peak time for myocardial ischaemia, ${ }^{12}$ acute myocardial infarction, ${ }^{34}$ and sudden cardiac death. ${ }^{56}$ Allied circadian patterns include morning peaks in heart rate, ${ }^{7}$ blood pressure, ${ }^{7}$ catecholamine and cortisol secretion, ${ }^{8}$ coronary vasoconstriction, ${ }^{9}$ platelet stickiness, ${ }^{10}$ and a morning trough in fibrinolytic activity. ${ }^{11} 12$ Studies have shown that $\beta$ blockade, by blunting the increase in the determinants of myocardial oxygen consumption, significantly altered the circadian pattern of ischaemia, ${ }^{1}$ myocardial infarction, ${ }^{34}$ and sudden cardiac death. ${ }^{13}$ The inference from these studies is that therapeutic protection should be maximal in the morning waking hours, and that at this time the determinants of myocardial oxygen consumption require suppression.

It is not clear whether the physiological pattern of heart rate alters appreciably in those who perform their primary activities in the evening or whether heart rate patterns are primarily related to the act of waking and are somewhat independent of the patterns of activity during daily life. To assess whether the circadian pattern of heart rate is significantly and reproducibly altered by work pattern we studied members of a symphony orchestra, who rose in the morning and were active during the day, before carrying out their major body of work in the evening.

We also assessed the cardiovascular responses of the orchestra during the rehearsals and the live performances and individual variations in response to different pieces of music. In view of the concern about "stress" in the players in an orchestra, we also investigated cardiovascular responses in members of the management and technical team for comparison.

Patients and methods

We studied 48 (36 men and 12 women aged 22-59 (mean 40)) members of the British Broadcasting Corporation Symphony Orchestra. All volunteered to undergo 24 hours of ambulatory ST segment monitoring on the day of a final rehearsal and live performance broadcast on radio 3 . Five were members of the management and technical staff of the orchestra. Instruments played during the monitoring periods included the violin (10), viola (three), cello (five), double bass (four), flute (four), trumpet (two), trombone (two), clarinet (two), bassoon (two), horn (four), oboe (one), tuba (one), organ (one), and tympani (two).

Thirty six subjects (35 players, 1 management) underwent 24 hours of electrocardiographic monitoring. This period included a final rehearsal in the morning and a live performance of Richard Strauss (Metamorphosen, Vier letze Lieder, and Die Frau ohne Schatten) and Mozart (Symphony No 40 in $G$ minor $(K 550)$ ) in the evening, 
performed at the Royal Festival Hall. Monitoring started before the rehearsal and the monitors were removed the next morning. Because some of the players thought that playing Strauss and Mozart was not particularly stressful we also monitored 21 people (17 players, four management staff) for 24 hours during final rehearsal and a live performance of Rachmaninov (Symphony No 2 in $E$ minor) and Tchaikovsky (Concert Fantasy: Bluebird pas de deux (Tchaikovsky/ Stravinsky)) at the Barbican Arts Centre. Nine (all musicians) had previously been monitored at the Royal Festival Hall. Again monitoring was started before the rehearsal and the monitors were removed the next morning.

We used pregelled electrodes to record two bipolar leads (an anterior lead (CM5) and an inferior lead). The sites and method of application were described elsewhere. ${ }^{14}$ Two channel recordings were taken on magnetic tape by an amplitude modulated dual channel recorder (Reynolds Tracker). All tapes were visually analysed at 60 times normal speed with a Reynolds Pathfinder 3 and printouts were at $25 \mathrm{~m} / \mathrm{s}$. Any rhythm disturbance or change in the ST segment was recorded. Significant ST segment depression was defined as planar or downsloping ST segment shift of $>1 \mathrm{~mm}$ measured $0.08 \mathrm{~s}$ after the $\mathrm{J}$ point that persisted for $>1$ minute.

One of the 48 individuals who underwent continuous ambulatory electrocardiographic monitoring was excluded from subsequent analysis because he was taking medications likely to affect the heart rate pattern. No other subject was taking any such medications at the time of study. Tapes from a further two subjects in the second concert (both also underwent monitoring during the first concert) were unanalysable for technical reasons.

In addition to visual analysis, we obtained a computerised printout of the results of each tape, which included the maximum and minimum heart rate recorded every five minutes throughout the 24 hour period of monitoring (total of 576 heart rate measurements/ person/24 h). A total of 32832 heart rate measurements from the analysable tapes were then manually incorporated on to a computerised worksheet for further analysis.

\section{STATISTICAL ANALYSIS}

Maximum and minimum heart rates for each five minute recording were averaged for the musicians monitored on the day of the first concert, those monitored on the day of the second concert, and those monitored on both concert days. The mean maximum and minimum heart rates were plotted against time of day to show the circadian distributions. Mean hourly maximum and minimum heart rates were also plotted and two harmonic Fourier fits superimposed to elucidate the circadian patterns. We used mean hourly heart rates to compare the circadian patterns with those previously described in healthy volunteers.

We used the Wilcoxon signed rank sum test to compare the mean maximum heart rates achieved by the musicians during both live performances and rehearsals; the hourly mean maximum heart rates of the musicians monitored on the day of concert 1 with those monitored on the day of concert 2; the heart rates of the string section during concert 1 with those of the wind section in the same concert; and the hourly mean maximal heart rates of the management and technical staff with those of the musicians. We used the Wilcoxon matched pairs rank sum test to compare heart rates achieved during concert 1 with those in concert 2 in those who performed live on both monitoring days.

\section{Results}

Data on 42 players (six in both concerts) and five members of the management and technical staff were available for analysis.

Figure 1 shows the circadian pattern of heart rate (mean maximum and minimum) in all 42 musicians investigated. This shows a peak in the morning waking hours (the recognised time of maximal heart rate activity in healthy people), but a greater peak during the evening. To establish whether the pattern of heart rate responses differed on the two concert days we compared the mean maximal and minimal heart rates of those studied every five minutes throughout the days (fig 2 ). This shows that the circadian patterns of mean maximal and minimal heart rates were similar on both monitoring days with a peak in heart rate in the

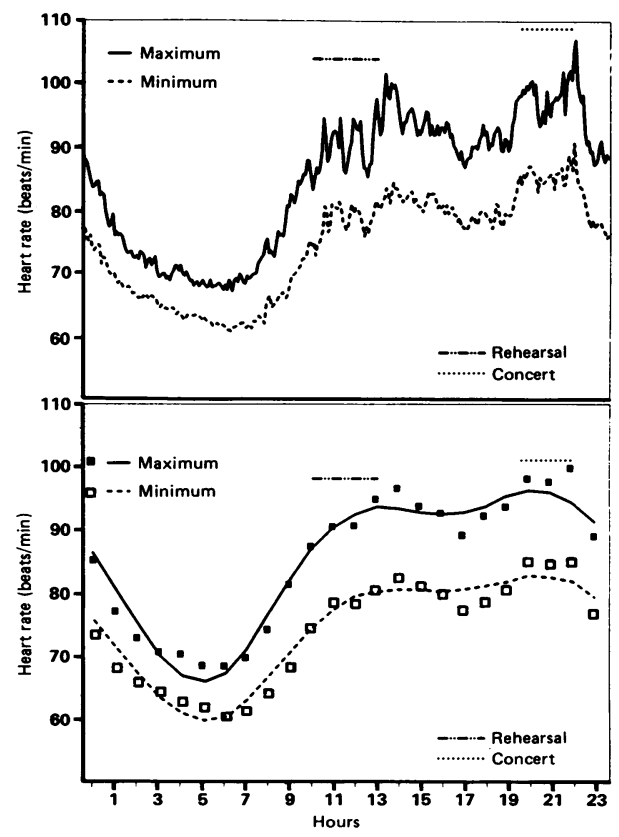

Figure 1 (Top) Circadian pattern of mean maximum and minimum heart rates measured every five minutes throughout the day in 42 players. (Bottom) Mean hourly maximum and minimum heart rates with bimodal fits superimposed. The approximate times of rehearsals and live performances are indicated. 
Figure 2 (Top) Circadian pattern of maximum and minimum heart rates measured every five minutes throughout the day in 34 players during 24 hours that included a concert (Strauss/Mozart). (Middle) Similar circadian pattern in 14 players throughout the day of the second concert (Rachmaninov) Tchaikovsky).

(Bottom) Fourier fits of mean maximum and minimum hourly heart rates of the two concert days.
Figure 3 (Top)

Circadian pattern of mean maximal heart rates in 42 musicians and 5 management/technical staff. (Bottom) Histogram showing the mean hourly differences in maximum heart rates between the management/technical staff and musicians.

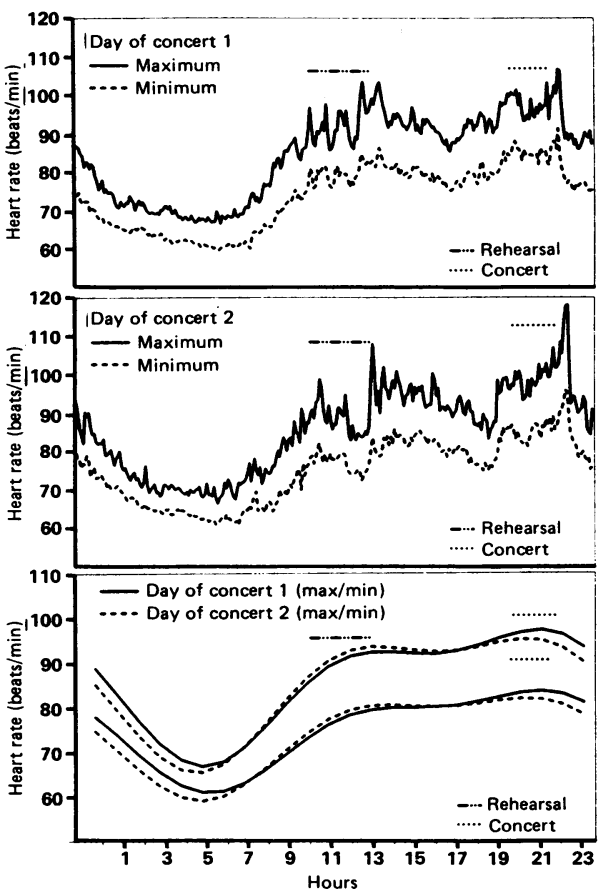

evening hours. Except for two hours $(13.00 \mathrm{~h}$ (97 beats/min concert $1 v 90$ beats/min concert $2, \mathrm{p}<0.05)$ and $22.00 \mathrm{~h}(97$ beats $/ \mathrm{min}$ concert $1 v 106$ beats/min concert $2, \mathrm{p}<0.05)$ ) there were no significant differences in hourly mean maximal heart rates during the two concert days.

Mean maximum heart rates were significantly higher during the live performances than during the final rehearsals (concert 1, 97.7 $v$

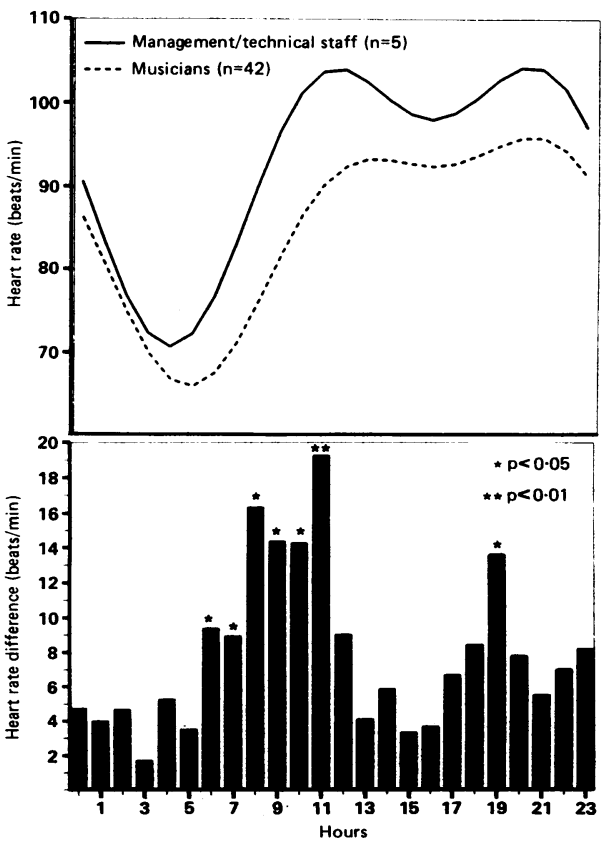

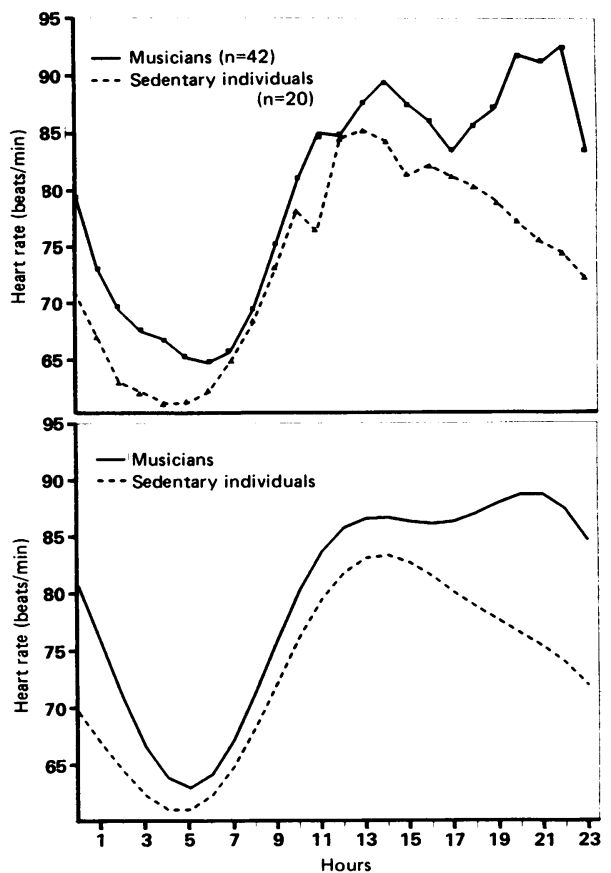

Figure 4 (Top) Comparison of the mean hourly heart rate changes in the 42 musicians during both monitoring days and the circadian pattern of mean hourly heart rates in 20 sedentary individuals. ${ }^{15}$ (Bottom) Fourier fits of mean hourly heart rates in 42 musicians and 20 sedentary individuals.

91.3 beats $/ \mathrm{min}, \mathrm{p}<0.05$ ) (concert $2,99.3 v$ 88.5 beats $/ \mathrm{min}, \mathrm{p}<0.05)$. Though there was no significant difference in the mean maximum heart rates achieved by the musicians during the two live performances (97.7 $v 99.3$ beats/ min), the six players (all wind and brass) who were monitored during both concerts had higher heart rates during Rachmaninov/ Tchaikovsky (Rachmaninov/Tchaikovsky 95.7 $v$ Strauss/Mozart 90.3 beats/min, $\mathrm{p}<0.05$ ). The mean maximal heart rate of the string section was significantly higher than that of the wind section during concert 1 , which included Metamorphosen by Strauss for 23 solo strings (string section $100.2 v$ wind section 91.8 beats/ $\min , \mathrm{p}<0.05)$.

We examined the mean hourly rate responses of the management and technical staff to establish whether the circadian pattern of heart rate responses differed in those who did not play an instrument. Figure 3 shows that the management and technical staff had a similar circadian pattern of heart rate to that of the musicians, with a primary peak in the evening hours. None the less, the management and technical staff had higher mean maximal heart rates throughout the day, and these differences were significant particularly in the periods leading up to the rehearsals and the live performances. Figure 4 shows the circadian pattern of heart rate in the $\mathbf{4 2}$ musicians monitored during the study compared with that of a group of 20 sedentary subjects described earlier. ${ }^{15}$ This shows that the circadian pattern of heart rate in 
the musicians was significantly altered at the times of the live performances.

Analysis of all tape results showed significant ST segment changes during the period of monitoring in one young subject $(2 \%)$. No significant arrhythmias were recorded during the monitoring periods.

\section{Discussion}

For many years there has been a debate as to whether the onset of end points of cardiovascular disease is related to physical activity. ${ }^{16}{ }^{17}$ Muller $e t$ al in a review of circadian variation and triggers of onset of acute cardiovascular disease concluded that daily activities trigger the onset of most cases of myocardial infarction and sudden cardiac death. ${ }^{18}$ There are distinctive circadian patterns of heart rate ${ }^{715}$ and of different disease processes, ${ }^{1-6}$ with a peak in the incidence of myocardial ischaemia, ${ }^{12}$ acute myocardial infarction,,$^{34}$ and sudden cardiac death ${ }^{56}$ in the morning waking hours. Though it is impossible to assume a cause and effect relation between myocardial ischaemia and the development of both acute myocardial infarction and sudden cardiac death on the basis of these observations, it has been suggested that they may be interrelated. ${ }^{19} 20$ The possible importance of changes in heart rate in the genesis of ischaemic episodes and various cardiovascular end points was suggested by the fact that whereas calcium antagonists with a coronary vasodilating capacity had no effect on the circadian pattern of ischaemia ${ }^{1}$ or myocardial infarction, ${ }^{4} \beta$ adrenergic blocking agents, which control heart rate and other determinants of myocardial oxygen consumption (thereby reducing myocardial oxygen demand), eliminated the distinctive morning peak of ischaemia, ${ }^{121}$ acute myocardial infarction, ${ }^{34}$ and sudden cardiac death. ${ }^{13}$ Many studies reported that $\beta$ blockade was effective in reducing the frequency of myocardial ischaemia, ${ }^{21-25}$ though the underlying pathophysiological mechanisms of ischaemia were not clarified. One of the conclusions from the studies of circadian variation is that antianginal medications should be given to offer maximal protection during the morning waking hours, the apparent time of greatest cardiovascular threat. ${ }^{1-6}$ Control of heart rate increase is of importance during this time.

To establish whether environmental factors do indeed significantly alter the recognised circadian pattern of heart rate, we studied a large group of patients who perform a normal day's work but whose major occupational efforts are in the evening. This distinguishes them from "shift workers" who maintain a normal sleep/wake/work pattern, despite having altered sleep/wake temporal patterns. We showed a considerable alteration in the circadian pattern of heart rate changes in such subjects, with the highest peak of activity in the evening hours, when most of their work was carried out, in addition to the early morning increase in heart rate as reported elsewhere. ${ }^{715}$ The explanation for this evening increase in activity is not merely that of playing an instrument in front of an audience, because the same pattern was found in members of the management and technical team associated with the orchestra. In previous studies on the circadian patterns of ischaemia, infarction, and sudden cardiac death ${ }^{135}$ a secondary evening peak in events was reported. This may have resulted simply from the fact that environmental circumstances lead to more cardiovascular stress in the evenings in some individuals, as shown in this study.

Northcote $e t$ al showed a circadian pattern of heart rate in individuals with a sedentary lifestyle that included some light activity. ${ }^{15}$ This resembled the pattern reported by MillarCraig et al in healthy individuals ${ }^{7}$ - that is a peak in heart rate activity in the morning waking hours and a gradual decline during the later hours. The pattern of heart rate changes was different in orchestral musicians, who work in the evenings as well as during more conventional hours, and this pattern of change was reproducible on different occasions.

Though the people we studied had no cardiovascular symptoms, individuals with asymptomatic or symptomatic coronary artery disease are also likely to have similar alterations in their circadian pattern of heart rate changes in response to environmental stress and therefore it is important to tailor medications to offer maximal protection, not necessarily in the morning, ${ }^{\prime}$ but at the times of identifiable cardiovascular stress during each patient's working day. We did not study the circadian patterns of blood pressure or catecholamine release in the present study; however, in earlier studies these variables peaked at the same time as heart rate changes, ${ }^{78}$ and they probably reflect cardiovascular stress in much the same way as heart rate changes do.

The increased heart rates during the live performances indicate the pressure of performing for an audience-in the evening the relative rise in heart rate from resting values is probably considerably greater than the rise recorded in the morning. Like Haider and Groll-Knapp in their study of the Vienna Symphony Orchestra, ${ }^{26}$ we too found that different pieces of music were associated with different levels of cardiovascular stress: though the overall changes in mean heart rate in both concerts were very similar, the heart rate change in the brass and wind players who were monitored during both concerts was greater during pieces by Rachmaninov and Tchaikovsky than during pieces by Strauss and Mozart.

The management and technical staff, who were responsible for ensuring the smooth running of the orchestra during performance days, had higher mean maximal heart rates than the players, mainly in the period leading up to the rehearsals and live performances. This accords with the theory that it is not the type of work that increases myocardial oxygen demand but the relative demands of getting the work done.

The lack of significant ST segment changes during ambulatory monitoring was somewhat surprising because a small but significant proportion of apparently healthy individuals had "ischaemic" ST segment changes during 
ambulatory monitoring. ${ }^{27} 28$ This finding does, however, confirm that screening seldom detects abnormalities in apparently healthy individuals, even during periods of pronounced cardiovascular stress. It also reflects the unique benefits of ambulatory monitoring for continuous cardiovascular assessment of large numbers of individuals-even during live recordings on national radio. Under these circumstances we had to use soundless machinery. The heart rate findings show that in occupations such as symphony orchestra members there is a relatively high level of stress throughout the day, reflecting what is a long day's work, though the nocturnal heart rate pattern resembled that previously reported for similar groups of healthy volunteers. ${ }^{715}$ To optimise treatment in patients with coronary artery disease the timing of occupational stress should be borne in mind.

We found that the circadian pattern of heart rate change was much influenced by environmental factors. The findings of previous studies, which identified the morning waking hours as the time of greatest cardiovascular danger, may in part have been the result of studying patients with normal working hours. The routine daily behavioural patterns of patients should be considered when tailoring treatment for optimal protection in patients with coronary artery disease. The pattern of heart rate (and probably blood pressure and catecholamine release) in those who work in the evening is likely to be different-with an increased risk of the end points of cardiovascular disease developing at this time. While it is not clear whether medical intervention to prevent possible triggers of end points of cardiovascular disease will reduce such episodes, it seems reasonable to assume that in patients with coronary artery disease optimal protection to minimise the physiological responses to stress will be beneficial. In the apparently healthy individuals that we studied, there was no evidence that any of the recorded cardiovascular responses were abnormal.

We would like to thank the Reynolds Medical Group for their cooperation in supplying the monitoring equipment for the periods of study, and the British Broadcasting Corporation periods of study, and the British Broadcasting Corporation
Symphony Orchestra members for taking part in the study.

1 Mulcahy D, Keegan J, Cunningham D, et al. Circadian variation of total ischsemic burden and its alteration with anti-anginal agents. Lancet 1988;ii:755-9.

2 Rocco MB, Barry J, Campbell S, et al. Circadian variation of transient myocardial ischaemia in patients with coronary artery disease. Circulation 1987;75:395-400.

3 Muller JE, Stone PH, Turi ZG, et al. Circadian variation in the frequency of onset of acute myocardial infarction. N Engl J Med 1985;313:1315-22.

4 Willich SN, Linderer $T$, Wegscheider $K$, Schroder $R$. Increased risk of myocardial infarction in the mornings [Abstract]. J Am Coll Cardiol 1988;11:28A.
5 Willich SN, Levy D, Rocco MB, Tofler GH, Stone PH, Muller JE. Circadian variation in the incidence of sudden cardiac death in the Framingham heart study population. Am J Cardiol 1987;60:801-6.

6 Muller JE, Ludmer PL, Willich SN, et al. Circadian variation in the frequency of sudden cardiac death Circulation 1987;75:131-8.

7 Millar-Craig MW, Bishop CN, Raftery EB. Circadian variation of blood pressure. Lancet 1978;1:795-7.

8 Turton NB, Deegan T. Circadian variation of plasma catecholamine, cortisol, and immunoreactive insulin concentrations in supine subjects. Clin Chim Acta 1974; 55:389-97.

9 Fugita M, Franklin D. Diurnal changes in coronary blood flow in conscious dogs. Circulation 1987;76:488-91.

10 Tofler GH, Brezinski D, Schafer AI, et al. Concurrent morning increase in platelet aggregability and the risk of myocardial infarction and sudden death. N Engl $J$ Med 1987;316:1514-8

11 Andreotti F, Khan MI, deBart A, et al. Circadian fluctuations in endothelium derived fibrinolytic factors in relation to the time of onset of myocardial infarction and sudden cardiac death [Abstract]. J Am Coll Cardiol 1988;11:27A

12 Angleton P, Chandler WL, Schmer G. Diurnal variation of tissue-type plasminogen activator and its rapid inhibitor (PAI-1). Circulation 1989;79:101-6.

13 Peters R, Muller JE, Goldstein S, Byington R, Friedman LM. Propranolol and the morning increase in the frequency of sudden cardiac

14 Quyyumi AA, Mockus L, Wright C, Fox KM. Morphology of ambulatory ST segment changes in patients with varying severity of coronary artery disease: investigation of the frequency of nocturnal ischaemia and coronary spasm. Br Heart J 1985;53:186-93.

15 Northcote RJ, Canning GP, Ballantyne D. Electrocardiographic findings in male veteran endurance athletes. Braphic findings in male $J$ 1989;61:155-60

16 Fitzhugh G, Hamilton BE. Coronary occlusion and fatal angina pectoris: study of the immediate causes and their angina pectoris: study of the immediate

17 Master AM. The role of effort and occupation (including physicians) in coronary occlusion. JAMA 1960;174: physician

18 Muller JE, Tofler GH, Stone PH. Circadian variation and triggers of onset of acute cardiovascular disease. Circulation 1989;79:733-43.

19 Assey ME, Walters GL, Hendrix GH, Carabello BA, Usher BW, Spann JF. Incidence of acute myocardial infarction in patients with exercise-induced silent myocardial ischaemia. Am J Cardiol 1987;59:497-500.

20 Sharma B, Asinger R, Francis GS, Hodges M, Wyeth RP. Demonstration of exercise induced painless myocardial ischemia in survivors of out-of-hospital vencula fibrillation. Am J Cardiol 1987;59:740-5.

21 Imperi GA, Lambert CR, Coy K, Lopez L, Pepine CJ. Effects of titrated beta blockade (metoprolol) on silent Effects of titrated beta blockade (metoprolol) on silent
myocardial ischaemia in ambulatory patients with coronmyocardial ischaemia in ambulatory patients with

22 Uusitalo A, Arstila M, Bae EA, et al. Metoprolol, nifedipine and combination in stable effort angina pectoris. $\mathrm{Am}$ Cardiol 1986;57:733-7.

23 Quyyumi AA, Crake T, Wright CM, Mockus LJ, Fox KM Medical treatment of patients with severe exertional and rest angina: double blind comparison of $\beta$ blocker, calcium antagonist, and nitrate. Br Heart $J$ 1987;57:505-11.

24 Willich SN, Pohjola-Sintonen S, Bhatia SJS, et al. Suppression of silent ischemia by metoprolol without alteration of morning increase of platelet agoregability in patients with stable corongry artery disease. Circulation 1989;79:557-65.

25 Egstrup $\mathrm{K}$. Randomised double blind comparison of metoprolol, nifedipine, and their combination in chronic metoprolol, nifedipine, and their combination in chronic stable angina: effects on total ischemic activity and heart

26 Haider M, Groll-Knapp E. Psychophysiological investigations into the stress experienced by musicians in a symphony orchestra. In: Braumuller $W$, ed. Stress and music. Medical, psychological, sociological, and legal strain factors in a symphony orchestra musician's profession. Vienna: Universitats-Verlagbuchhandlung, 1981

27 Quyyumi AA, Wright C, Fox K. Ambulatory electrocardiographic ST segment changes in healthy volunteers. Br Heart J 1983;50:460-4.

28 Kohli RS, Cashman PMM, Lahiri A, Raftery EB. The ST segment of the ambulatory electrocardiogram in a normal population. Br Heart J 1988;60:4-16. 